\title{
Modeling of Reactive Ion Etching for $\mathrm{Si} / \mathrm{SiO}_{2}$ Systems
}

\author{
S. Hamaguchi and H. Ohta \\ Department of Fundamental Energy Science, Kyoto University, \\ Uji, Kyoto 611-0011.
}

\begin{abstract}
Molecular dynamics (MD) simulations have been used to study surface reaction dynamics of $\mathrm{Si}$ and $\mathrm{SiO}_{2}$ etching by halogens. To perform classical MD simulations for $\mathrm{SiO}_{2}$ etching reaction by halogens $(\mathrm{Cl}$ or $\mathrm{F})$, we have constructed new sets of two- and three-body interatomic potential functions based on potential energy data obtained from $a b$ initio quantum mechanical calculations of the electronic states. Etching yields for $\mathrm{Si}$ and $\mathrm{SiO}_{2}$ targets are obtained from MD simulations for both beam etching and reactive ion etching (RIE). The obtained yields are in good agreement with experimental observations.
\end{abstract}

\section{Introduction}

Patterned etching of $\mathrm{Si}$ is one of the most important fabrication processes employed in the semiconductor chip manufacturing. To transfer desired submicron patterns to the Si surface efficiently, one needs to use highly selective etching processes. As the minimum dimensions of required etched patterns are now close to $0.1 \mu$ under the current state-of-the-art manufacturing technologies and are diminishing rapidly, development of more reliable, high-throughput processes with good critical-dimension control is pursued aggressively in the semiconductor industry.

One of the most important issues for the control of critical dimensions in such processes is to understand the surface reaction mechanisms during actual processing conditions. In the present paper, we focus on $\mathrm{Si}$ and $\mathrm{SiO}_{2}$ etching processes by plasmas containing halogen ( $\mathrm{Cl}$ or $\mathrm{F}$ ) atoms. Using molecular dynamics (MD) simulations, we attempt to elucidate details of etching processes under realistic etching conditions.

Classical MD simulations with empirical potentials have been used by various authors to analyze details of surface reaction dynamics [1]-[9]. Data obtained from such simulations can compensate for scarcity of some experimental data. As to beam etching and/or reactive ion etching (RIE) of Si by halogens, StillingerWeber (SW) type interatomic potentials $[1,2]$ are known to represent many aspects of surface reactions during etching processes with reasonable accuracy.

To extend such MD simulations to etching of $\mathrm{SiO}_{2}$ surfaces by halogens, we have developed new potential sets for Si-O-F and $\mathrm{Si}-\mathrm{O}-\mathrm{Cl}$ systems [10], which are consistent with previously known interatomic potentials of SW type for $\mathrm{Si}$ 
[1], Si-Cl [2], Si-F [3], and Si-O [11] systems. As the SW-type potential functions include three-body interactions, we have determined ternary interactions by fitting a functional form to potential energy data obtained from ab initio calculations of electronic states of such systems.

\section{Molecular Dynamics Simulation}

The simulation method is now presented briefly. Atoms are normally injected to a target which is placed in a simulation cell with periodic boundary conditions imposed in the horizontal directions. The atoms in the bottom layer are rigidly fixed to prevent the drift of the entire simulation cell. Since energetic ions impinging on the surface are expected to be neutralized near the target surface due to an Auger emission process, we only consider charge-neutral atoms as impinging species. It is also assumed that the target surface is kept chargeneutral during the process.

After injection of each atom, we let the system evolve for 0.7 ps under the constant total-energy conditions. Most transient processes such as rapid release of the kinetic energy of the injected atom to the target are almost completed during this period. We then artificially cool the entire system (by decreasing the velocities of all atoms) for $0.3 \mathrm{ps}$ to reduce the target temperature to the initial temperature (i.e., $300^{\circ} \mathrm{K}$ ) in order to prevent the system from being unrealistically heated up.

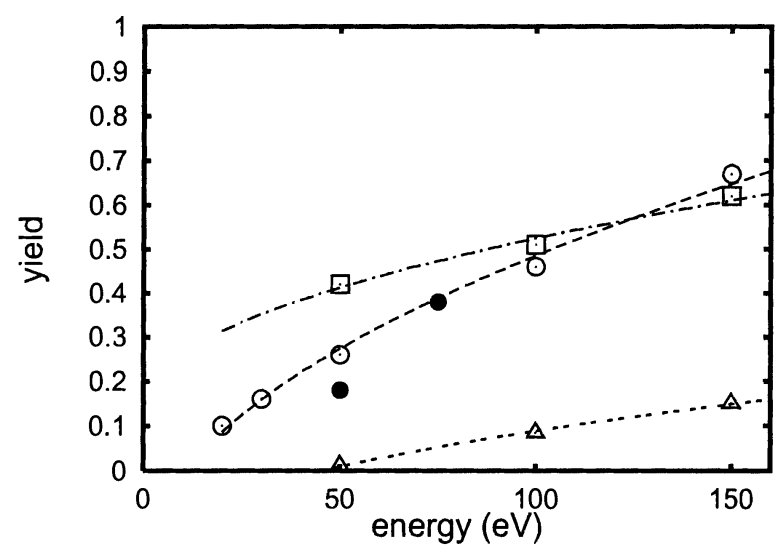

Fig. 1. Etching yields of the silicon target for $\mathrm{Cl}(\bigcirc), \mathrm{F}(\square)$, and $\operatorname{Ar}(\triangle)$ beam etching. The filled circles represent etching yields for a $\mathrm{Cl}$ RIE process with Ar bombardment. 


\section{Conclusions}

We have performed $\mathrm{MD}$ simulations of $\mathrm{Si}$ and $\mathrm{SiO}_{2}$ etching by $\mathrm{Cl}$ and $\mathrm{F}$ monomer beams as well as Ar beams with various impinging energies. Etching yields obtained from our MD simulations are shown in Figs. 1 and 2. Here Si etching yields (i.e., the number of $\mathrm{Si}$ atoms emitted from the surface per impact) that we have obtained are in good agreement with experimental observations and previous $\mathrm{MD}$ simulation results [9]. There are not many experimentally obtained $\mathrm{SiO}_{2}$ etching yields by $\mathrm{Cl}$ or $\mathrm{F}$ monoenergetic beams, with which we could compare our simulation results. However our observation of the threshold energy for $\mathrm{SiO}_{2}$ etching by Ar impact (which is about $50 \mathrm{eV}$ ) is in good agreement with earlier experimental observations $[12,13]$.

We have also simulated RIE processes. The RIE simulation results are indicated by filled circles in Figs. 1 and 2. Here we have injected about $100 \mathrm{Cl}$ atoms with low kinetic energy $(1 \mathrm{eV})$ into the target surface in each interval between two successive Ar injections. As shown in both Figs. 1 and 2, the etching yields by $\mathrm{Ar}$ impact increase up to those by $\mathrm{Cl}$ beam etching since the low energy $\mathrm{Cl}$ atoms cover the target surface and terminate $\mathrm{Si}$ and $\mathrm{O}$ bonds.

In summary, we have performed MD simulations to study surface reactions during $\mathrm{Si}$ and $\mathrm{SiO}_{2}$ etching by halogens. The obtained results are consistent with various experimental observations that have been obtained to date.

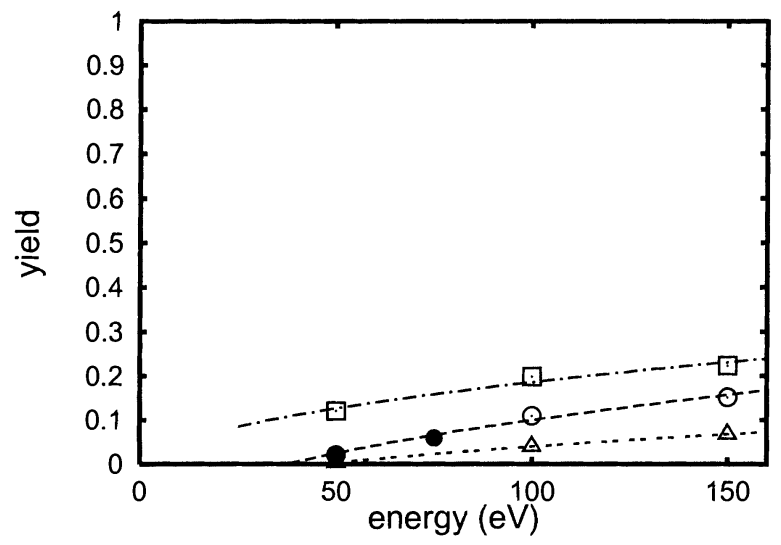

Fig. 2. Etching yields of the $\mathrm{SiO}_{2}$ target for $\mathrm{Cl}(\mathrm{O}), \mathrm{F}(\square)$, and $\operatorname{Ar}(\triangle)$ beam etching. The filled circles represent etching yields for a $\mathrm{Cl}$ RIE process with Ar bombardment. 


\section{References}

[1] F. H. Stillinger and T. A. Weber, Phys. Rev. B 31, 5262 (1985); J. Chem. Phys. 88, 5123 (1988); Phys. Rev. Lett. 62, 2144 (1989).

[2] T. A. Weber and F. H. Stillinger, J. Chem. Phys. 926239 (1990).

[3] H. Feil, J. Dieleman, and B. J. Garrison, J. Appl. Phys. 74, 1303 (1993).

[4] R. Smith, D. E. Harrison, Jr., and B. J. Garrison, Phys. Rev. B 40, 93 (1989).

[5] T. A. Schoolcraft and B. J. Garrison, J. Vac. Sci. Technol. A8, 3496 (1990); J. Am. Chem. Soc. 113, 8221 (1991).

[6] M. E. Barone and D. B. Graves, J. Appl. Phys. 77,1263 (1995); J. Appl. Phys. 78,6604 (1995).

[7] D. E. Hanson, A. F. Voter, and J. D. Kress, J. Appl. Phys. 82, 3552, 1997.

[8] N. A. Kubota and D. J. Economou, S. J. Plimpton, J. Appl. Phys. 83, 4055, 1998.

[9] H. Ohta and S. Hamaguchi, submitted to J. Vac. Sci. Technol. A (2001).

[10] H. Ohta and S. Hamaguchi, submitted to J. Chem. Phys. (2001).

[11] T. Watanabe, H. Fujiwara, H. Noguchi, T. Hosono, and I. Ohdomari, Jpn. J. App. Phys. L38, 366 (1999).

[12] W. M. Holber and J. Forster, in Proceeding of Symposium on Dry Process, (ed. by Y. Horiike et al. The Institute of Electrical Engineers of Japan, Technical Committee on Electron Devices, Tokyo) p. 9 (1989).

[13] D. J. Oostra, R. P. van Ingen, A. Haring, A. E. de Vries, and G. N. A van Veen, Appl. Phys. Lett. 501506 (1987) 